\title{
Novel Treatments of Psychosis
}

\author{
Walter Dunn • Stephen R. Marder
}

Published online: 28 January 2015

(C) Springer International Publishing AG (outside the USA) 2015

\begin{abstract}
Since the advent of chlorpromazine in the 1950s, $\mathrm{D}_{2}$ receptor antagonist activity has been synonymous with antipsychotic medications. This single mechanism of action may explain the similarities in efficacy among approved antipsychotics. Additionally, extrapyramidal and metabolic side effects of antipsychotics present a significant burden to patient quality of life. Development of the next generation of antipsychotics has focused on novel mechanisms of action including drugs that act through the N-methyl-D-aspartate (NMDA) system, modify second messenger activity, and modulate neuronal firing. This review surveys a selection of novel treatments with activity against positive symptoms. Each section includes a brief description of their mechanism of action, summarizes studies evaluating their clinical efficacy, and comments on safety and tolerability issues. Included in this review are compounds originally developed for treating psychotic disorders such as pimavanserin, pomaglumetad, and bitopertin. Other interventions are off-label uses of existing treatments such as bexarotene, rTMS, and sodium nitroprusside.
\end{abstract}

Keywords Antipsychotics · Transcranial direct current stimulation · Psychosis · Psychotic symptoms · Positive symptoms $\cdot$ NMDA $\cdot$ Schizophrenia $\cdot$ Glycine transporter

This article is part of the Topical Collection on Psychosis

\author{
W. Dunn $\cdot$ S. R. Marder \\ Semel Institute for Neuroscience at UCLA, 11301 Wilshire Blvd, Bld \\ 210a, Rm 130, Los Angeles, CA 90073, USA \\ W. Dunn \\ e-mail: wdunn@mednet.ucla.edu \\ W. Dunn $\cdot$ S. R. Marder $(\square)$ \\ VA Desert Pacific Mental Illness, Research, Education, and Clinical \\ Center, 11301 Wilshire Blvd, Bld 210a, Rm 130, Los \\ Angeles, CA 90073, USA \\ e-mail: SMarder@mednet.ucla.edu
}

inhibitor · Transcranial magnetic stimulation · Metabotropic glutamate receptor $\cdot$ Novel treatments

\section{Introduction}

The first effective medication for psychosis - chlorpromazine-was introduced in the 1950s. Since then, scores of antipsychotics have been developed and introduced into clinical care. The effects of these agents on psychotic symptoms are related to their affinity for $\mathrm{D}_{2}$ dopamine receptors where they are antagonists. This single mechanism of action probably explains why all of these medications - with the exception of clozapine - have similar efficacy. The finding that clozapine had superior efficacy to other dopamine antagonists led to a search for other agents with similar efficacy. Unfortunately, the group of dopamine antagonists developed after clozapine did not achieve this superior efficacy. As a result, drug discovery for psychosis has focused on targets other than dopamine receptors.

This review on novel treatments for psychosis will focus mostly on approaches that are not related to $\mathrm{D}_{2}$ antagonism. None of these treatments are currently approved for use in schizophrenia or other psychotic disorders. In addition, this review is not meant to be exhaustive. Rather, we focus on a number of drugs and devices that appear promising. Although there has been substantial activity in drug development of cognitive impairment in schizophrenia and negative symptoms, this review focuses only on drugs that address psychotic symptoms.

\section{NMDA System}

The N-methyl-D-aspartate (NMDA) receptor has emerged as an attractive target for drug development in schizophrenia. 
This interest stems from the observation that administration of NMDA receptor antagonists, such as phencyclidine (PCP) and ketamine, can cause schizophrenia-type symptoms - including psychosis, cognitive impairments, and negative symptoms - in subjects without schizophrenia [1-7]. This suggests that schizophrenia may be associated with NMDA receptor hypofunction, and that enhancing NMDA activity may be a treatment for schizophrenia [3].

The ionotropic NMDA receptor is composed of multiple subunits including GluN1 and GluN2, and occasionally GluN3 subunits [8-10]. Receptor activation requires the excitatory neurotransmitter glutamate, in addition to binding of a co-agonist, glycine, at the allosteric modulator site. Compounds that act at this allosteric modulator site have been identified and show promise in treating the positive psychotic symptoms of schizophrenia [11•].

\section{Glycine Transporter Inhibitors}

Drugs can tonically activate NMDA receptor activity by increasing occupancy at the allosteric modulator site [12-14]. One target of these drugs is the glycine transporter (GlyT-1) which is responsible for the re-uptake of glycine in the synaptic cleft. Similar to the action of serotonin re-uptake inhibitors on serotonin, GlyT-1 inhibitors increase the synaptic concentration of glycine resulting in supraphysiological receptor occupancy. Increasing occupancy at the allosteric modulator site is thought to compensate for general NMDA receptor deficits. One compound with this activity is sarcosine, which is a naturally occurring metabolite from which glycine is synthesized. Lane et al., a group from Taiwan, conducted several clinical studies demonstrating symptom reduction with sarcosine monotherapy and combination therapy with non-clozapine antipsychotics [15]. In 2008, they reported the results of a 6week trial involving acutely symptomatic schizophrenia patients treated with sarcosine monotherapy ( 1 vs. 2 g/day). Although there was no significant effect of sarcosine dose, they demonstrated that patients with no prior antipsychotic exposure were more likely to respond to the $2 \mathrm{~g} /$ day regimen [12].

In 2010, Lane et al. published their latest sarcosine results from a 6-week placebo-controlled, add-on trial of sarcosine and D-serine in chronic schizophrenia patients. Consistent with their earlier pilot studies, sarcosine added to antipsychotics was superior to antipsychotics alone. The outcomes for sarcosine were superior to that of D-serine but not statistically significant. Intra-group effect sizes between the Positive and Negative Symptom Scale (PANSS) total end-point and baseline scores were the largest for sarcosine (1.10), followed by D-serine (0.86), and placebo (0.17) [16].

A 2011 meta-analysis of NMDA receptor modulators for treatment of schizophrenia determined an overall medium effect size of sarcosine on total schizophrenia symptoms (positive, negative, and cognitive domains). The effect size on positive symptoms, however, was small and did not reach statistical significance [11•]. The advantages of sarcosine are that it is safe, well tolerated, and causes minimal adverse effects both in monotherapy and add-on treatment (added to non-clozapine antipsychotics) $[12,15,16]$.

Bitopertin, also a GlyT-1 inhibitor, had been under development by Roche for the treatment of negative and positive symptoms. Six phase III trials were initiated based on a promising proof of concept study. The positive results of this proof of concept study were published in 2014; however, the subsequent phase III trials yielded less encouraging results $[17,18]$. As of April 2014, all phase III trials with the exception of Nightlyte, an evaluation of bitopertin for sub-optimally controlled symptoms of schizophrenia (positive symptoms), had been terminated $[19,20]$.

Metabotropic Glutamate Receptor Agonists

In addition to NMDA ionotropic glutamate receptors, metabotropic glutamate receptors (mGlu) have also been identified as potential targets for treating schizophrenia. Specifically, group II mGlu receptors (mGlu2 and mGlu3) moderate glutamate release, preventing glutamate toxicity. These receptors are expressed in the prefrontal cortex, hippocampus, and amygdala, which are involved in the pathophysiology of schizophrenia [21, 22].

Pomaglumetad was developed as an agonist to mGlu2/3 receptors. Activation of these receptors is proposed to normalize the hyperactivated cortical glutamate state secondary to NMDA receptor hypofunction [23, 24].

The initial proof of concept study reported promising results with pomaglumetad separating from placebo on PANSS total score. It was also superior to the active control in the trial, olanzapine, in terms of weight gain. Pomaglumetad-treated patients lost a mean of $0.51 \mathrm{~kg}$ after 4 weeks of treatment [24]. Unfortunately, these positive results were followed by a single failed and two negative trials.

The subsequent phase II trial was designed to examine efficacy over a larger dose range in acutely exacerbated schizophrenia patients. The trial did not achieve interpretable results, however, as both pomaglumetad and the active control, olanzapine, failed to separate from placebo due to an unusually high placebo response [25]. An additional phase II trial examined pomaglumetad as a monotherapy in acute psychotic exacerbations in schizophrenia. In this trial, pomaglumetad failed to separate from placebo while the active control, risperidone, did demonstrate superiority over placebo [26].

Based on the negative results of the phase II studies, Eli Lilly stopped their program for pomaglumetad in 2012 [24]. Although the commercial development of pomaglumetad for schizophrenia is currently inactive, the data suggest that drugs with similar mGlu2/3 agonist mechanisms may be preferable 
over existing antipsychotics. These agonists have a more favorable safety profile. Unlike current treatments, pomaglumetad was well tolerated and had a low incidence of extrapyramidal symptoms (EPS), hyperprolactinemia, and weight gain [24, 25, 27, 28].

\section{Second Messenger Activity}

First described in the 1800 s, sodium nitroprusside is presently used in the ICU setting for treatment of hypertensive urgency/ emergency. Its mechanism of action is the generation of nitric oxide leading to blood vessel dilation and lowering of blood pressure [29]. One proposed pathway of psychosis in schizophrenia is the dysregulation of the glutamate nitric oxide cyclic guanosine monophosphate (cGMP) network. Decreased levels of nitric oxide metabolites and cGMP have been found in patients with schizophrenia compared to healthy controls $[30,31]$. Thus, the generation of nitric oxide and cGMP by sodium nitroprusside may alleviate these deficiencies, leading to a resolution of associated psychotic symptoms [32••].

An alternative mechanism of action involves modulation of NMDA receptor activity. In vitro assays have demonstrated that sodium nitroprusside blocks $\mathrm{Ca}^{2+}$ currents into the cell, and thus potentially inhibits NMDA receptor activation [33, 34].

Hallak et al. conducted a randomized, double-blind, placebocontrolled trial from 2007 to 2009 involving 20 patients with schizophrenia admitted for acute psychotic episodes. These subjects were randomized to receive placebo or a single infusion of low-dose $(0.5 \mathrm{ug} / \mathrm{kg} / \mathrm{min}$ for $4 \mathrm{~h})$ sodium nitroprusside at the time of admission. Comparatively, average doses for treatment of hypertensive emergency are in the range of $3-4 \mathrm{ug} / \mathrm{kg} / \mathrm{min}$. In this study, patients demonstrated a rapid response to this single $4 \mathrm{~h}$ infusion by the second hour of administration. Improvement was seen in the overall BPRS-18 total score with the activation subscale and thinking disorder scores demonstrating the most rapid response to treatment. After this single dose, continued symptom response was reported out to 4 weeks [32••].

Intriguing aspects of this study are the rapid and sustained improvement of symptoms after a single administration of sodium nitroprusside. Despite the encouraging findings, limitations of the study include the small number of subjects and permission of medication changes $48 \mathrm{~h}$ after administration of sodium nitroprusside. Thus, symptom improvement beyond $48 \mathrm{~h}$ cannot be solely attributed to sodium nitroprusside treatment [32••].

\section{Serotonin System}

\section{5- $\mathrm{HT}_{2 \mathrm{~A}}$ Antagonists}

One characteristic of newer antipsychotics that distinguishes them from older drugs is their inverse agonist activity at the 5-
$\mathrm{HT}_{2 \mathrm{~A}}$ receptor. Inverse agonists bind to receptors that have constitutive activity independent of ligand binding and antagonize that constitutive activity $[35,36]$. Pimavanserin is a compound developed by ACADIA Pharmaceuticals that acts as an inverse agonist at the $5-\mathrm{HT}_{2 \mathrm{~A}}$ receptor. It has nearly $100-$ fold more selectivity at this receptor than to $5-\mathrm{HT}_{2 \mathrm{~B}}, 5-\mathrm{HT}_{2 \mathrm{C}}$, and $5-\mathrm{HT}_{6}$, and no activity at the $\mathrm{D}_{2}$ receptor. This unique binding profile likely accounts for the lack of motor and metabolic side effects which are commonly seen with existing antipsychotics [37, 38].

Currently, pimavanserin is being developed for the primary indication of Parkinson's disease psychosis (PDP), where $\mathrm{D}_{2}$ receptor antagonism is undesired due to potential worsening of motor symptoms. In patients with PDP, pimavanserin at $40 \mathrm{mg}$ demonstrated clinically meaningful improvement over placebo in phase II trials on measures of psychosis on a modified SAPS-PD scale. Importantly, pimavanserin did not worsen motor symptoms [38, 39].

Pimavanserin is also being studied for the treatment of schizophrenia as an adjunctive to low sub-therapeutic doses of antipsychotics. This polypharmacy combination is proposed to minimize metabolic and EPS side effects while achieving comparable psychotic symptom reduction [40••]. In a double-blind, 6-week trial, 423 chronic schizophrenia patients with acute relapses were recruited. They were treated with a combination of pimavanserin $20 \mathrm{mg}$ added to doses of risperidone ( $2 \mathrm{mg}$ ) or haloperidol $(2 \mathrm{mg}$ ) considered to be subtherapeutic. These combinations were compared to the same doses ( $2 \mathrm{mg}$ ) of the two antipsychotics alone, and to a standard therapeutic dose of risperidone $(6 \mathrm{mg})$. Low-dose risperidone (2 mg)+ pimavanserin performed better than low-dose risperidone alone with statistically superior measures on the PANSS total score, PANSS Negative Symptom, and General Psychopathology subscales. A similar outcome was seen with the positive symptom subscale at the trend level $(p=0.058)$. In comparison to risperidone $6 \mathrm{mg}$ alone, the low-dose combination achieved equal symptom control but with less weight gain and prolactin elevation. Interestingly, the treatment arm of haloperidol $(2 \mathrm{mg}$ ) alone performed as well as the risperidone $6 \mathrm{mg}$ treatment and combination haloperidol+ pimavanserin, suggesting that haloperidol at the $2 \mathrm{mg}$ dose is effective in treating acute exacerbations in chronic schizophrenia patients. Although the additional $5-\mathrm{HT}_{2 \mathrm{~A}}$ blockade by pimavanserin did not enhance the efficacy of haloperidol, it also did not increase the side effect burden or adversely affect tolerability $[40 \bullet \bullet]$.

Currently, pimavanserin is in phase II development for Alzheimer's disease psychosis and an additional study for the treatment of schizophrenia is planned [38]. Given the positive data for PDP, a FDA new drug application (NDA) for pimavanserin as a PDP treatment will be filed in the near future [41]. See Table 1 for the proposed timeline of the NDA filing. 
Table 1 Overview of novel treatments for psychosis

\begin{tabular}{|c|c|c|c|}
\hline Treatment & Mechanism & Developmental status & Notes \\
\hline Sarcosine & GlyT-1 inhibitor & Phase II & No active trials ${ }^{\mathrm{a}}$ \\
\hline Bitopertin & GlyT-1 inhibitor & Phase III & $\begin{array}{l}\text { Development for schizophrenia } \\
\text { discontinued by Roche }\end{array}$ \\
\hline Pomaglumetad & mGlu2/3 agonist & Phase II & Development discontinued by Eli Lilly \\
\hline Sodium nitroprusside & Nitric oxide generation & $\begin{array}{l}\text { FDA approved for hypertensive } \\
\text { emergency/urgency }\end{array}$ & Single published study [34] \\
\hline Pimavanserin & $\begin{array}{l}5-\mathrm{HT}_{2 \mathrm{~A}} \text { inverse } \\
\text { agonist }\end{array}$ & Phase III & NDA likely to be filed for $\mathrm{PDP}^{\mathrm{b}}$ in 2105 \\
\hline Bexarotene & Retinoid $\mathrm{X}$ receptor agonist & FDA approved for T cell lymphoma & Augmentation of antipsychotic treatmen \\
\hline Cariprazine & $\begin{array}{l}\mathrm{D}_{3} / \mathrm{D}_{2} \text { receptor partial } \\
\text { agonist }\end{array}$ & Phase III & Monotherapy \\
\hline PF-02545920 & PDE10A inhibitor & Phase II & No active trials for schizophrenia \\
\hline TAK-063 & PDE10A inhibitor & Phase I & \\
\hline $\begin{array}{l}\text { Transcranial direct current } \\
\text { stimulation (tDCS) }\end{array}$ & Neuromodulation & Pre-clinical & \\
\hline $\begin{array}{l}\text { Repetitive transcranial magnetic } \\
\text { stimulation (rTMS) }\end{array}$ & Neuromodulation & $\begin{array}{l}\text { FDA approved for treatment } \\
\text { of depression }\end{array}$ & $\begin{array}{l}\text { Off-label treatment of auditory } \\
\text { hallucinations }\end{array}$ \\
\hline
\end{tabular}

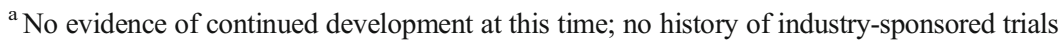

${ }^{\mathrm{b}}$ Parkinson's disease psychosis

\section{Retinoid Signaling Pathway}

The retinoid signaling pathway is a complex metabolic system responsible for the growth, differentiation, and function of cells. It has also been implicated in neural development and homeostasis in vertebrates. Briefly, retinoids bind to retinoid $\mathrm{X}$ receptors which then regulate the expression of specific genes. Dysfunction of this pathway, either through toxicity or deficit of retinoic acid, leads to a constellation of symptoms including cognitive impairment, enlarged ventricles, and developmental abnormalities [42, 43].

Goodman first presented the retinoid dysregulation hypothesis by proposing three lines of evidence implicating its involvement in the pathogenesis of schizophrenia [44]. First, toxicity or deficiency of retinoic acid causes developmental abnormalities. This includes cognitive impairment, similar to that seen in schizophrenia patients and their family members. Second, genetic loci that are associated with schizophrenia contain genes in the retinoid cascade. Third, retinoic acid regulates the transcriptional activation of the $\mathrm{D}_{2}$ receptor, the target of current antipsychotic medications [44].

\section{Retinoid X Receptor Agonist}

There is evidence that retinoid transporters and synthesis of retinoic acid is decreased in schizophrenia patients compared to healthy controls $[42,43]$. Based on these lines of evidence, Lerner et al. proposed using a retinoid $\mathrm{X}$ receptor agonist to augment antipsychotics to treat patients with schizophrenia [45]. Bexarotene is a retinoid that selectively binds retinoid $\mathrm{X}$ receptors and is FDA approved for the treatment of cutaneous T cell lymphoma. It is typically administered in the 300-600 $\mathrm{mg}$ range and adverse effects include hypothyroidism and dyslipidemia. Lerner et al. established the safety and tolerability of bexarotene in an open-label pilot study with a 75-mg dose added to standard antipsychotic therapy. In this 6 week study of 25 patients, improvement was observed on subscales of the PANSS including the total Positive and Negative Scale Score, general psychopathology, positive, and dysphoric mood factor scores. Adverse events included an increase in total serum cholesterol beyond the normal range, and a decrease in thyroid stimulating hormone (TSH) that remained within normal limits [45]. In a subsequent 6-week, double-blind, randomized placebo-controlled trial, bexarotene $75 \mathrm{mg}$ was added to 79 patients with schizophrenia or schizoaffective disorder on stable regimens of antipsychotics. The subjects demonstrated a moderate improvement $(d=0.48, p=0.003)$ on the PANSS positive scale in week 6 scores compared to baseline. In contrast to the pilot study, the change in general psychopathology and dysphoric mood subscales did not reach significance. Similar adverse events were observed in this study as there was an increase in total cholesterol and decrease in total $\mathrm{T}_{4}$. An additional finding was that patients not taking lipid-lowering agents responded most robustly to the treatment, demonstrating the greatest improvement on the PANSS positive scale score [46].

One advantage of bexarotene is that it is an FDA-approved medication already in use for other off-label indications (lung and breast cancer). While potentially promising as an add-on therapy, the lipid raising effect of bexarotene may be a limitation. Given the adverse metabolic effects of second generation 
antipsychotics, patients may be taking statins, which could interfere with bexarotene.

\section{Dopamine Hypothesis}

Despite intense interest in alternative models such as the NMDA system to explain the pathogenesis of schizophrenia, the dopamine hypothesis continues to form the basis for many antipsychotic drugs in development. In addition to antagonist activity at the $\mathrm{D}_{2}$ receptor, however, these next generation of agents act as partial agonists, target $\mathrm{D}_{3}$ receptors, and are active at serotonin receptors [14, 47-49].

\section{Dopamine Receptor Blockade}

Cariprazine has partial agonist activity at $\mathrm{D}_{3}$ and $\mathrm{D}_{2}$ receptors with higher affinity for $\mathrm{D}_{3}$. It is currently in late stage clinical development. Phase II and phase III trials have investigated its use in schizophrenia, bipolar disorder, and unipolar depression $[50,51]$. The FDA issued a complete response letter in November 2013 indicating that the completed trials had demonstrated the effectiveness of cariprazine in treating schizophrenia, but that given the complex pharmacokinetics and metabolism of the compound, additional clinical trial data was needed to determine optimal dosing parameters [52].

\section{Phosphodiesterase Inhibitors}

Manipulation of the dopamine system and its direct and indirect pathways has been explored through modulation of upstream second messenger molecules. Specifically, phosphodiesterase 10A (PDE10A) regulates intracellular second messengers cAMP and cGMP by inactivating them. Inhibition of PDE10A is proposed to increase levels of these molecules and activate cAMP/PKA signaling in the indirect pathway. This is thought to inhibit the thalamocortical circuits thereby mimicking $\mathrm{D}_{2}$ antagonism $[53,54]$. In direct pathway neurons, the activation of cAMP/PKA signaling is thought to reproduce $\mathrm{D}_{1}$ agonist activity [54].

Papaverine, an opium alkaloid, was originally studied for its PDE10a inhibitory activity. Its use, however, was limited for clinical applications given its poor potency and short halflife after systemic administration [53]. New PDE10A inhibitors with more favorable characteristics are in various stages of development. Pfizer's compound, PF-02545920, was listed as a phase II drug for adjunctive treatment in schizophrenia and for Huntington's disease in their Aug 2014 company pipeline announcement [55]. As of December 2014, however, the ClinicalTrials.gov registry listed only one completed phase II trial, and several other phase II studies for this compound in subjects with schizophrenia had been terminated [56]. Takeda
Pharmaceutical's PDE10A inhibitor, TAK-063, is currently listed as a Phase I drug in the company's October 2014 drug pipeline announcement. Two completed phase I studies are reported for TAK-063 in the ClinicalTrials.gov registry, but there are currently no listings of trials actively recruiting for patients $[57,58]$.

\section{Neuromodulation}

Neuromodulation involves the alteration of neuronal or nerve activity through administration of electrical activity or chemical agents to specific sites in the body. In neuropsychiatric disorders, non-invasive brain stimulation methods have become increasingly popular given their targeted approach, minimal side effects, and limited drug interactions. Currently, there are no FDA-approved neuromodulation systems for the treatment of schizophrenia [59-64]. Off-label use of approved devices, however, has demonstrated promising results, especially in treating the positive symptoms of schizophrenia.

\section{Transcranial Direct Current Stimulation}

Transcranial direct current stimulation (tDCS) is a noninvasive neuromodulation technique that administers direct electrical current to targeted brain regions. The essential components of tDCS are a constant current generator and two electrodes. Direct current passes from the anode to cathode electrode, going through the scalp and cortex at strengths ranging from 1 to $2 \mathrm{~mA}$ for $10-30 \mathrm{~min}$ [61].

The basic premise of tDCS is that neurons beneath the anode electrode are depolarized, bringing them closer to their firing threshold. Conversely, cathodal stimulation hyperpolarizes neurons making them less likely to fire. Unlike transcranial magnetic stimulation (TMS), tDCS does not produce neuronal firing. Rather, tDCS modulates neurons so that they are more likely or less likely to fire. Therefore, in neuropsychiatric disorders where particular brain regions or networks exhibit diminished activity, anodal stimulation would be used to increase activity in these hypofunctioning regions. Conversely, cathodal stimulation could modulate hyperactive regions [65-68].

The changes induced by tDCS extend beyond the active stimulation period. The effects from treatment have been observed for as long as $90 \mathrm{~min}$ past a single stimulation [61]. Studies involving multiple stimulations have demonstrated effects lasting several months after the treatment [59, 69••]. The mechanisms responsible for the long-term effects of tDCS have not been fully elucidated, but there is evidence supporting involvement of the NMDA system, long-term potentiation, and GABAergic interneurons [61]. 
tDCS has been applied in a variety of settings ranging from stroke rehabilitation to neurocognitive enhancement [61]. In 2012, Brunelin et al. described a robust finding using tDCS to treat 30 schizophrenia patients with refractory auditory hallucinations. This trial was a randomized, double-blind, shamcontrolled design with 15 patients receiving active treatment and the remaining undergoing sham stimulation. They targeted the tempo-parietal cortex with inhibitory cathodal tDCS, resulting in a mean $31 \%$ decrease in the AHRS Score assessing auditory hallucinations, $d=1.58$. Stimulations were delivered for $20 \mathrm{~min}$ at $2 \mathrm{~mA}$, twice a day for five consecutive weekdays for a total of ten treatments. In addition to an acute decrease in auditory hallucinations, there was an improvement in the total PANSS score $(d=0.98, p=0.01)$, especially in the negative symptom domain $(d=1.07, p=0.01)$. Beyond the acute response to treatment, the reduction of auditory hallucinations lasted 3 months post-treatment [69・•].

The primary advantages of tDCS are its low cost, ease of use, and minimal side effects. tDCS is presently not FDA approved for any neuropsychiatric indications. Constant direct current generators are widely available, however, and tDCS is increasing being studied in patient populations.

\section{Repetitive Transcranial Magnetic Stimulation}

Repetitive transcranial magnetic stimulation or rTMS is a neuromodulatory technique that induces or suppresses neuronal activity through electrical currents generated by a magnetic field. High-frequency rTMS $(5-10 \mathrm{~Hz})$ is believed to increase cortical activity, whereas low-frequency $(1 \mathrm{~Hz})$ treatments are proposed to suppress neuronal firing. The strategy for treating psychiatric illnesses targets hypoactive brain regions with high-frequency rTMS in order to "restore" normal activity. Overactive brain regions are targeted with lowfrequency rTMS to reduce neuronal firing to normal levels $[64,70]$.

Already FDA approved for the treatment of unipolar depression, rTMS has been shown in studies to have promise in treating psychotic symptoms associated with schizophrenia.

Of these studies, those that have used low-frequency rTMS to treat auditory hallucinations refractory to standard antipsychotic management have demonstrated the most encouraging results. Several groups have hypothesized that an overactive left temporal-parietal cortex (TPC) is one of the regions responsible for auditory hallucinations, and have targeted this area with $1 \mathrm{~Hz}$ rTMS $[63,71]$. These groups have treated up to 50 subjects with rTMS for 4-9 days, resulting in a reduction of auditory hallucinations [63]. The Hoffman et al. studies from 2003 to 2005 were double-blinded, randomized, shamcontrolled trials demonstrating a positive response to lowfrequency rTMS. One of the major strengths of these studies was the use of an active coil tilted 45 deg away from the scalp in the sham condition. This replicated the sound and somatic sensation associated with an active treatment while minimizing stimulation voltages in the brain to one third of that in the active condition [71, 72]. The Brunelin et al. study was also double-blinded, randomized, and sham controlled, but they used a non-active coil for their sham condition. This inactive coil reproduced the sound of stimulation but not the somatic sensation of active stimulation [68].

There has also been lack of response to rTMS stimulation in some studies. However, these negative studies treated patients for a shorter duration of time, suggesting that inadequate dosing accounted for the negative findings. Additional treatment considerations include regions targeted and coil-tocortex distance. Although the literature has been mixed in regards to rTMS treatment of positive symptoms, the most robust findings have been with low-frequency rTMS of the TPC. Such treatments reduced auditory hallucinations with durations of effect ranging from 10 to 15 weeks after 12 weeks of stimulation [63].

Advantages of rTMS include its few side effects and the existing FDA-approved indication for the treatment of unipolar depression. Given the recent proliferation of rTMS units in the community, the infrastructure exists for rTMS treatment of psychotic symptoms to be logistically feasible.

\section{Conclusion}

With the exception of pimavanserin, we are not aware of non$\mathrm{D}_{2}$ antagonist treatments for psychosis that are approved or near approval at this time. Other than low-frequency rTMS for refractory auditory hallucinations, which is currently in offlabel use, nearly all of the approaches described in this review are in relatively early stages of development. Pomaglumetad and bitopertin did reach later phase evaluation but were not found to be effective. Improving psychosis treatment is an important goal and there are a number of promising approaches that may eventually reach the clinics.

Acknowledgments WD receives fellowship support through the VA Desert Pacific Mental Illness Research, Education, and Clinical Center.

Stephen R. Marder, MD, receives support from the VA Desert Pacific Mental Illness Research, Education, and Clinical Center.

\section{Compliance with Ethics Guidelines}

Conflict of Interest WD has no conflicts of interest to report. SRM was a paid consultant to the following companies: Abbvie, Otsuka, Pfizer, Merck, Roche, Genentech, Forum, Targacept, Lundbeck, Takeda, and Forest. He received research support from Sunovion, Amgen, Genentech, Psychogenics, and Synchroneuron.

Human and Animal Rights and Informed Consent This article does not contain any studies with human or animal subjects performed by the authors. 


\section{References}

Papers of particular interest, published recently, have been highlighted as:

- Of importance

•- Of major importance

1. Steullet, P., et al., Redox dysregulation, neuroinflammation, and NMDA receptor hypofunction: a "central hub" in schizophrenia pathophysiology? Schizophr Res, 2014.

2. Lindsley $\mathrm{CW}$ et al. Progress towards validating the NMDA receptor hypofunction hypothesis of schizophrenia. Curr Top Med Chem. 2006;6(8):771-85.

3. Kantrowitz J, Javitt DC. Glutamatergic transmission in schizophrenia: from basic research to clinical practice. Curr Opin Psychiatry. 2012;25(2):96-102.

4. Allen RM, Young SJ. Phencyclidine-induced psychosis. Am J Psychiatry. 1978;135(9):1081-4.

5. Jodo E. The role of the hippocampo-prefrontal cortex system in phencyclidine-induced psychosis: a model for schizophrenia. J Physiol Paris. 2013;107(6):434-40.

6. Becker A, Grecksch G. Ketamine-induced changes in rat behaviour: a possible animal model of schizophrenia. Test of predictive validity. Prog Neuropsychopharmacol Biol Psychiatry. 2004;28(8): 1267-77.

7. Andersen JD, Pouzet B. Spatial memory deficits induced by perinatal treatment of rats with PCP and reversal effect of D-serine. Neuropsychopharmacology. 2004;29(6):1080-90.

8. Zhu S, Paoletti P. Allosteric modulators of NMDA receptors: multiple sites and mechanisms. Curr Opin Pharmacol. 2014;20C:14-23.

9. Paoletti P, Bellone C, Zhou Q. NMDA receptor subunit diversity: impact on receptor properties, synaptic plasticity and disease. Nat Rev Neurosci. 2013;14(6):383-400.

10. Paoletti P. Molecular basis of NMDA receptor functional diversity. Eur J Neurosci. 2011;33(8):1351-65.

11. Singh SP, Singh V. Meta-analysis of the efficacy of adjunctive NMDA receptor modulators in chronic schizophrenia. CNS Drugs. 2011;25(10):859-85. A meta-analysis of treatments that act modulate NMDA receptor activity for treatment of chronic schizophrenia. The review summarizes individual studies and provides effect sizes reported in the studies.

12. Lane HY et al. Sarcosine (N-methylglycine) treatment for acute schizophrenia: a randomized, double-blind study. Biol Psychiatry. 2008;63(1):9-12.

13. Hashimoto K. Glycine transporter-1: a new potential therapeutic target for schizophrenia. Curr Pharm Des. 2011;17(2):112-20.

14. Miyamoto $\mathrm{S}$ et al. Treatments for schizophrenia: a critical review of pharmacology and mechanisms of action of antipsychotic drugs. Mol Psychiatry. 2005;10(1):79-104.

15. Lane HY et al. Sarcosine or D-serine add-on treatment for acute exacerbation of schizophrenia: a randomized, double-blind, placebo-controlled study. Arch Gen Psychiatry. 2005;62(11): 1196-204.

16. Lane $\mathrm{HY}$ et al. A randomized, double-blind, placebo-controlled comparison study of sarcosine (N-methylglycine) and D-serine add-on treatment for schizophrenia. Int $\mathbf{J}$ Neuropsychopharmacol. 2010;13(4):451-60.

17. Goff DC. Bitopertin: the good news and bad news. JAMA Psychiatry. 2014;71(6):621-2.

18. Umbricht $\mathrm{D}$ et al. Effect of bitopertin, a glycine reuptake inhibitor, on negative symptoms of schizophrenia: a randomized, double-blind, proof-of-concept study. JAMA Psychiatry. 2014;71(6):637-46.
19. Roche. Investor Update 15 April 2014. . 2014 December 4, 2014]; Available from: http://www.roche.com/inv-update-2014-04-15-e.pdf.

20. ClinicalTrials.gov. Search of: RO4917838-List ResultsClinicaltrials.gov. December 5, 2014; Available from: http:// www.clinicaltrials.gov/ct2/results?term=RO4917838\&Search= Search.

21. Marek GJ et al. Glutamatergic (N-methyl-D-aspartate receptor) hypofrontality in schizophrenia: too little juice or a miswired brain? Mol Pharmacol. 2010;77(3):317-26.

22. Tsai G, Coyle JT. Glutamatergic mechanisms in schizophrenia Annu Rev Pharmacol Toxicol. 2002;42:165-79.

23. Liu $\mathrm{W}$ et al. Pharmacogenetic analysis of the mGlu2/3 agonist LY2140023 monohydrate in the treatment of schizophrenia. Pharmacogenomics J. 2012;12(3):246-54.

24. Kinon BJ, Gomez JC. Clinical development of pomaglumetad methionil: a non-dopaminergic treatment for schizophrenia. Neuropharmacology. 2013;66:82-6.

25. Kinon BJ et al. A multicenter, inpatient, phase 2, double-blind, placebo-controlled dose-ranging study of LY2140023 monohydrate in patients with DSM-IV schizophrenia. J Clin Psychopharmacol. 2011;31(3):349-55.

26. Gomez, B.K.B.A.M.A.C.D.L.Z.V.L.S.S.W.A.J.C. LY2140023 Monohydrate in the treatment of patients with Schizophrenia: results of 2 clinical trials assessing efficacy in treating acutely ill patients and those with prominent negative symptoms. in 14th International Congress on Schizophrenia Research 2013. Grande Lakes.

27. Adams DH et al. Pomaglumetad methionil (LY2140023 monohydrate) and aripiprazole in patients with schizophrenia: a phase 3, multicenter, double-blind comparison. Schizophr Res Treat. 2014;2014:758212.

28. Adams DH et al. A long-term, phase 2, multicenter, randomized, open-label, comparative safety study of pomaglumetad methionil (LY2140023 monohydrate) versus atypical antipsychotic standard of care in patients with schizophrenia. BMC Psychiatry. 2013;13:143.

29. Hottinger DG et al. Sodium nitroprusside in 2014: a clinical concepts review. J Anaesthesiol Clin Pharmacol. 2014;30(4):462-71.

30. Ramirez $\mathrm{J}$ et al. Low concentration of nitrite and nitrate in the cerebrospinal fluid from schizophrenic patients: a pilot study. Schizophr Res. 2004;68(2-3):357-61.

31. Gattaz WF, Cramer H, Beckmann H. Low CSF concentrations of cyclic GMP in schizophrenia. Br J Psychiatry. 1983;142:288-91.

32.• Hallak JE et al. Rapid improvement of acute schizophrenia symptoms after intravenous sodium nitroprusside: a randomized, doubleblind, placebo-controlled trial. JAMA Psychiatry. 2013;70(7):66876. This is the first placebo controlled study to demonstrate that sodium nitroprusside has antipsychotic activity in a schizophrenia population in the setting of an acute exacerbation of symptoms.

33. Manzoni $\mathrm{O}$ et al. Sodium nitroprusside blocks NMDA receptors via formation of ferrocyanide ions. Neuroreport. 1992;3(1):77-80.

34. Hoyt KR et al. Nitric oxide modulates NMDA-induced increases in intracellular $\mathrm{Ca} 2+$ in cultured rat forebrain neurons. Brain Res. 1992;592(1-2):310-6.

35. Aloyo VJ et al. Current status of inverse agonism at serotonin2A (5HT2A) and 5-HT2C receptors. Pharmacol Ther. 2009;121(2):160 73.

36. Abbas A, Roth BL. Pimavanserin tartrate: a 5-HT2A inverse agonist with potential for treating various neuropsychiatric disorders. Expert Opin Pharmacother. 2008;9(18):3251-9.

37. Meltzer HY, Roth BL. Lorcaserin and pimavanserin: emerging selectivity of serotonin receptor subtype-targeted drugs. J Clin Invest. 2013;123(12):4986-91.

38. Hacksell $U$ et al. On the discovery and development of pimavanserin: a novel drug candidate for Parkinson's psychosis. Neurochem Res. 2014;39(10):2008-17. 
39. Cummings J et al. Pimavanserin for patients with Parkinson's disease psychosis: a randomised, placebo-controlled phase 3 trial. Lancet. 2014;383(9916):533-40.

40.• Meltzer HY et al. Pimavanserin, a selective serotonin (5-HT)2Ainverse agonist, enhances the efficacy and safety of risperidone, $2 \mathrm{mg}$ /day, but does not enhance efficacy of haloperidol, $2 \mathrm{mg} /$ day: comparison with reference dose risperidone, 6mg/day. Schizophr Res. 2012;141(2-3):144-52. This is the first published study demonstrating antipsychotic activity in a schizophrenia population. This study also found that therapeutic doses of haloperidol are likely lower than has been traditionally used in the clinical setting.

41. WIre, B. ACADIA pharmaceuticals announces expedited path to NDA filing for pimavanserin following meeting with FDA. 2014 [cited 2014 December, 5]; Available from: http://www. businesswire.com/news/home/20130411005398/en/ACADIAPharmaceuticals-Announces-Expedited-Path-NDA-Filing\#. VIGa0Kkmjtg.

42. Citver AS et al. Indirect modulation of dopamine D2 receptors as potential pharmacotherapy for schizophrenia: III. Retinoids. J Clin Pharm Ther. 2002;27(3):161-8.

43. Goodman AB. Microarray results suggest altered transport and lowered synthesis of retinoic acid in schizophrenia. Mol Psychiatry. 2005;10(7):620-1.

44. Goodman AB. Three independent lines of evidence suggest retinoids as causal to schizophrenia. Proc Natl Acad Sci U S A. 1998;95(13):7240-4.

45. Lerner $\mathrm{V}$ et al. Bexarotene as add-on to antipsychotic treatment in schizophrenia patients: a pilot open-label trial. Clin Neuropharmacol. 2008;31(1):25-33.

46. Lerner $\mathrm{V}$ et al. The retinoid $\mathrm{X}$ receptor agonist bexarotene relieves positive symptoms of schizophrenia: a 6-week, randomized, double-blind, placebo-controlled multicenter trial. J Clin Psychiatry. 2013;74(12):1224-32.

47. Davis KL et al. Dopamine in schizophrenia: a review and reconceptualization. Am J Psychiatry. 1991;148(11):1474-86.

48. Seeman P. Dopamine receptor sequences. Therapeutic levels of neuroleptics occupy D2 receptors, clozapine occupies D4. Neuropsychopharmacology. 1992;7(4):261-84.

49. Miyamoto $\mathrm{S}$ et al. Pharmacological treatment of schizophrenia: a critical review of the pharmacology and clinical effects of current and future therapeutic agents. Mol Psychiatry. 2012;17(12):1206-27.

50. Veselinovic T, Paulzen M, Grunder G. Cariprazine, a new, orally active dopamine D2/3 receptor partial agonist for the treatment of schizophrenia, bipolar mania and depression. Expert Rev Neurother. 2013;13(11):1141-59.

51. Citrome L. Cariprazine in schizophrenia: clinical efficacy, tolerability, and place in therapy. Adv Ther. 2013;30(2):114-26.

52. Forest Laboratories, I. Forest Laboratories and Gedeon Richter receive complete response letter for cariprazine. December 4, 2014; Available from: http://investor.frx.com/press-release/r-and-d-news/ forest-laboratories-and-gedeon-richter-receive-complete-responseletter-c.

53. Schmidt CJ et al. Preclinical characterization of selective phosphodiesterase 10A inhibitors: a new therapeutic approach to the treatment of schizophrenia. J Pharmacol Exp Ther. 2008;325(2):681-90.

54. Kehler J, Nielsen J. PDE10A inhibitors: novel therapeutic drugs for schizophrenia. Curr Pharm Des. 2011;17(2):137-50
55. Pfizer. Pfizer Pipeline as of August 7, 2014. December 5, 2014; Available from: http://www.pfizer.com/sites/default/files/productpipeline/8 7 2014 Pipeline Update.pdf.

56. ClinicalTrials.gov. Search of: PF-02545920- List ResultsClinicalTrials.gov. December 5, 2014; Available from: http:/ www.clinicaltrials.gov/ct2/results?term=PF-02545920\&Search= Search.

57. Limited, T.P.C. Pipeline As of October 30, 2014. December 5, 2014; Available from: http://www.takeda.com/research/files/ pipeline_20141030_en.pdf.

58. ClinicalTrials.gov. Search of: TAK-063-List Results-Clinical Trials.gov. 2014 [cited 2014 December 5]; Available from: http:// www.clinicaltrials.gov/ct2/results?term=TAK-063\&Search= Search.

59. Andrade C. Once- to twice-daily, 3-year domiciliary maintenance transcranial direct current stimulation for severe, disabling, clozapine-refractory continuous auditory hallucinations in schizophrenia. J ECT. 2013;29(3):239-42.

60. Shiozawa $\mathrm{P}$ et al. Transcranial direct current stimulation (tDCS) for the treatment of persistent visual and auditory hallucinations in schizophrenia: a case study. Brain Stimul. 2013;6(5):831-3.

61. Nitsche MA et al. Transcranial direct current stimulation: state of the art 2008. Brain Stimul. 2008;1(3):206-23.

62. Sommer IE et al. The treatment of hallucinations in schizophrenia spectrum disorders. Schizophr Bull. 2012;38(4):704-14.

63. Stanford AD et al. rTMS strategies for the study and treatment of schizophrenia: a review. Int J Neuropsychopharmacol. 2008;11(4): $563-76$

64. Kim DR, Pesiridou A, O'Reardon JP. Transcranial magnetic stimulation in the treatment of psychiatric disorders. Curr Psychiat Rep. 2009;11(6):447-52

65. Aleman A, Sommer IE, Kahn RS. Efficacy of slow repetitive transcranial magnetic stimulation in the treatment of resistant auditory hallucinations in schizophrenia: a meta-analysis. J Clin Psychiatry. 2007;68(3):416-21.

66. Hajak $\mathrm{G}$ et al. High-frequency repetitive transcranial magnetic stimulation in schizophrenia: a combined treatment and neuroimaging study. Psychol Med. 2004;34(7):1157-63.

67. Holi MM et al. Left prefrontal repetitive transcranial magnetic stimulation in schizophrenia. Schizophr Bull. 2004;30(2):429-34.

68. Brunelin $\mathrm{J}$ et al. Low frequency repetitive transcranial magnetic stimulation improves source monitoring deficit in hallucinating patients with schizophrenia. Schizophr Res. 2006;81(1):41-5.

$69 . \bullet$ Brunelin $\mathrm{J}$ et al. Examining transcranial direct-current stimulation (tDCS) as a treatment for hallucinations in schizophrenia. Am J Psychiatry. 2012;169(7):719-24. This is the first controlled studies to demonstrate that tDCS can be successfully used in a schizophrenia population and has activity against psychotic symptoms.

70. Lopez-Ibor JJ, Lopez-Ibor MI, Pastrana JI. Transcranial magnetic stimulation. Curr Opin Psychiatry. 2008;21(6):640-4.

71. Hoffman RE et al. Temporoparietal transcranial magnetic stimulation for auditory hallucinations: safety, efficacy and moderators in a fifty patient sample. Biol Psychiatry. 2005;58(2):97-104.

72. Hoffman RE et al. Transcranial magnetic stimulation of left temporoparietal cortex and medication-resistant auditory hallucinations. Arch Gen Psychiatry. 2003;60(1):49-56. 